\title{
Which Beta-Blocker is Most Effective in Heart Failure?
}

\author{
Willem J. Remme
}

Published online: 2 July 2010

(C) Springer Science+Business Media, LLC 2010

\section{Introduction}

Since the mid-late nineties beta-blockade has become one of the pillars of chronic heart failure treatment. Based on a significant and consistent beneficial effect on survival and a reduction of worsening heart failure (HF) in large, placebocontrolled studies performed at that time, beta-blockade has been accepted as mandatory therapy in patients with stable mild, moderate and severe HF. These pivotal studies were, in order of sequence: the US carvedilol programme (carvedilol), CIBIS II (bisoprolol), MERIT-HF (metoprolol), and COPERNICUS (carvedilol) performed in patients with chronic HF and left ventricular (LV) dysfunction [1-4]. Whereas the focus in the first 3 studies was in mild/ moderate HF, COPERNICUS was exclusively carried out in patients with severe, albeit stable. HF. In addition, in patients with LV dysfunction and/or HF directly after an acute myocardial infarction, carvedilol also significantly improved survival compared to placebo treatment [5]. A later study, SENIORS, performed in an elderly population irrespective of cardiac function with nebivolol, showed a significant reduction in the primary outcome, a composite of all-cause mortality and hospital admission for HF, but for all-cause mortality alone [6]. In all of these studies background therapy included ACE inhibitors.

As a consequence of these results, carvedilol, bisoprolol, metoprolol succinate and nebivolol have been accepted for the treatment of chronic, stable HF (NYHA II-IV) of ischemic or non-ischemic origin in international guidelines for the treatment of HF [7, 8].

W. J. Remme ( $\square)$

Sticares Cardiovascular Research Institute, P.O. Box 882, 3160 AB Rhoon, The Netherlands

e-mail: w.j.remme@sticares.org
Such guidelines however do not address an important question for the clinician: "which beta-blocker to use"? Are they all similarly effective and equally tolerated, or may there be a preferred beta-blocker for the treatment of chronic heart failure?

\section{Pharmacological properties of beta-blocking drugs}

The beta-blockers accepted for HF treatment differ considerably (Table 1). Metoprolol and bisoprolol are beta-1 selective agents. Nebivolol is also a beta-1 selective drug, but has additional vasodilating properties, possibly related to endothelial nitric oxide generation.

In contrast, carvedilol blocks not only the beta-1 receptor, but also the beta- 2 and alpha- 1 adrenoceptors. In addition, carvedilol has anti-oxidative and anti-endothelin properties.

The question arises whether the different adrenergic blocking activities of these beta-blockers are relevant to their beneficial effect in heart failure. One important direction to answer that question without the need for large controlled studies concerns their individual modulating effect on cardiac remodeling [9].

\section{Beta-blockade and cardiac remodeling}

Various mechanisms contribute to cardiac remodeling, including neurohormonal and cytokine activation, inflammation, activation of inducible NO synthase, oxidative stress, apoptosis and ischemia, as well as mechanical factors such as stretch and activation of mechano-sensitive ion channels [10]. These factors do not operate in isolation, but often interrelate. Stretch activates neurohormonal pathways including the renin angiotensin system and the alpha1 adrenergic system [11]. Sympathetic overactivation may augment cytokine production, which in turn increases 
Table 1 Properties of selected beta-blockers

${ }^{a}$ Anti-oxidant, anti-apoptotic, inhibit endothelin \# NO generation

\begin{tabular}{llllll}
\hline & $\beta_{1}$ blockade & $\beta_{2}$ blockade blockade & $\alpha_{1}$ blockade & ISA & Ancillary effects $^{\text {a }}$ \\
\hline Carvedilol & +++ & +++ & +++ & - & $+++^{\mathrm{a}}$ \\
Metoprolol & +++ & - & - & - & - \\
Bisoprolol & +++ & - & - & - & - \\
Bucindolol & +++ & +++ & +++ & +++ & - \\
Nebivolol & +++ & - & - & - & $++\#$ \\
\hline
\end{tabular}

inducible NO $[12,13]$. The excess NO may then lead to oxyradical formation, oxidative stress and apoptosis. This complex of interactions indicates the potential usefulness of adrenergic blockade, besides other forms of neurohormonal blockade, in modulating cardiac remodeling.

Sympathetic blockade may be particularly important in modulating remodeling for several reasons. Activation of the sympathetic system occurs early after a cardiac insult leading to remodeling, possibly earlier than other neurohormonal systems [14]. Also, in heart failure the cardiac sympathetic tone is excessively high. Sufficiently so that cardiac catecholamine levels become toxic for cardiomyocytes, and may result in cardiac necrosis $[15,16]$. Desensitization of beta-receptors, but also beta-blockade may counteract these effects [17].

Also, high catecholamine levels may induce apoptosis. Beta-blockade counteracts this by increasing anti-apoptotic factors, such as Bcl-2 and $\mathrm{Bcl} \mathrm{X}(\mathrm{S})$, and by reducing those which are pro-apoptotic (e.g. Bax) [18].

A further mechanism through which beta-blockade may affect remodeling concerns its effect on cardiac metabolism. Sympathetic activation in heart failure promotes the use of free fatty acids instead of carbohydrates for energy production. This means less ATP produced for the same amount of available oxygen. Beta-blockade counteracts this waste of ATP by increasing the dependency on carbohydrates, thus improving ATP availability in the energystarved failing heart.

Finally, beta-blockers reduce heart rate, which is beneficial in reducing cardiac oxygen consumption, while at the same time, by lengthening the diastolic period, improving oxygen supply. Lengthening of the diastolic period also allows more time for the uptake of calcium by the sarcoplasmatic reticulum, possibly improving cardiac relaxation.

\section{Anti-remodeling properties in heart failure: are all beta- blockers equally effective?}

Blockade of all adrenergic receptors should be preferable to selective beta-blocking agents for modulation of cardiac remodeling. Not only the beta-1 receptor, but also the beta2 and alpha-1 receptor are linked to downstream cellular signaling pathways resulting in cardiac remodeling. Of importance, these pathways are not similar. Consequently, blocking only the beta-1 receptor, leaves the heart unprotected to remodeling signals triggered by stimulation of the other adrenergic receptors (Fig. 1). Myocardial stretch, an important factor in remodeling, is a good example. It increases alpha-adrenergic activation, which may result in cardiac hypertrophy and induce toxic effects on the heart. Furthermore, alpha-adrenergic stimulation may lead to peripheral (including renal) and coronary vasoconstriction with potentially harmful effects, including increased afterload, renal hypoperfusion and myocardial ischemia. Blockade of alpha-adrenergic receptors therefore appears a logical approach in heart failure and would support the use of carvedilol rather than selective beta-adrenergic blockers.

Beta-2 receptor activation facilitates norepinephrine release from the heart through stimulation of pre-synaptic cardiac beta- 2 receptors. In addition, beta -2 stimulation may promote cardiac fibrosis and lead to arrhythmias [19, 20]. Whether these negative effects are offset by the positive effects of beta-2 stimulation on apoptosis is questionable. As beta-2 receptors are percent-wise increased in heart failure, blocking their effects appears a logical step in heart failure treatment and would again favour carvedilol to selective beta-1 blocking drugs, such as metoprolol and bisoprolol. In atrial muscle from patients with non-terminal heart failure, carvedilol was a 13 -fold more potent antagonist of the effect of adrenaline on beta- 2

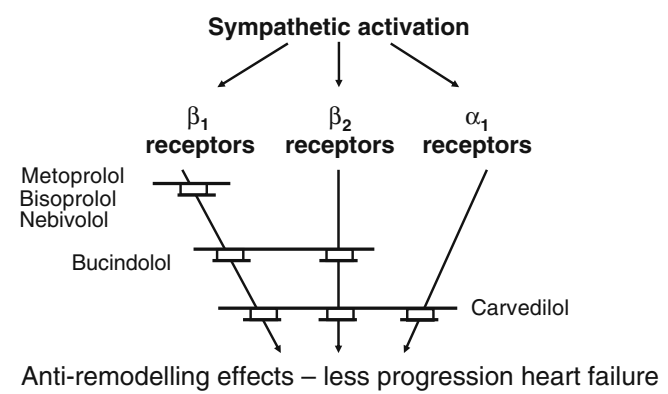

Fig. 1 Schematic representation of adrenergic receptors and blockade by selective beta-1 blockers (metoprolol, bisoprolol, nebivolol, beta-1 and -2 blockers (propranolol, bucindolol) and the beta-1 and -2 blocker and alpha- 1 adrenergic blocker carvedilol. The latter exerts the most protective effect against cardiac remodeling by blocking all 3 receptors 
receptors than that of noradrenaline on beta- 1 receptors [21]. In the same study, carvedilol, but not metoprolol, reduced the sensitivity of atrial muscle to adrenaline and noradrenaline in cardiac tissue from terminal hear failure patients.

Arguably, of the three adrenergic receptors, the beta- 1 receptor is probably the most important in regards sympathetic stimulation of cardiac remodeling. This would support specific beta-1 receptor blockade. However, carvedilol, compared to metoprolol, has a tighter binding to the beta-1 receptor and, hence, a longer-lasting effect [22].

Moreover, carvedilol reduces the increased expression of the beta-3 adrenergic receptor in heart failure in contrast to metoprolol, with a more effective improvement of hemodynamics and a greater attenuation of cardiac remodeling [23]. Furthermore, carvedilol stimulates beta-arrestin signaling, a unique mechanism, compared to other betablockers, which could contribute to its efficacy in heart failure therapy [24].

\section{Carvedilol as preferred beta-blocker in cardiac remodeling and heart failure-preclinical arguments}

Carvedilol protects better against cardiac remodeling and subsequent heart failure than more selective beta-adrenergic blockers. The arguments for this includes its comprehensive blocking of all 3 adrenergic receptors, as indicated above, and its anti-oxidant, anti-apoptotic and anti-endothelin effects.

Compared to metoprolol succinate, carvedilol at dosages that led to comparable heart rate reductions, induced significantly greater improvements of cardiac function in dogs with pacing-induced heart failure, and increased renal, hepatic, and skeletal muscle blood flow [25]. In addition, it improved myocardial glucose uptake (providing better energy conservation) compared to metoprolol, and antagonized the response to exogenous norepinephrine to a greater extent than metoprolol succinate.

In rodents with isoproterenol-induced cardiac hypertrophy and heart failure, carvedilol resulted in a greater reduction of heart weight and increase in cAMP than with metoprolol, despite similar reductions in heart rate [26]. Comparison of the R-and S-enantiomeres of carvedilol further indicated that the reduction in heart weight resulted from alpha-1 blockade and that beta-2 blockade contributed to the improvement of cAMP.

The importance of alpha-adrenergic blockade in remodeling was further indicated in studies by Lai et al, who observed that carvedilol, but not metoprolol or propranolol, suppressed collagen production in norepinephrine-stimulated cardiac fibroblasts [27]. Similar suppressing effects were found with doxasozine, an alpha-blocking agent.

In the presence of increased sympathetic tone carvedilol, but not metoprolol, may provide angiotensin-1 receptor blockade through its alpha-1 adrenergic blocking properties, comparable to the effects of angiotensin receptor blockers, inhibiting the vasoconstrictor effect of angiotensin II on human coronary micro-arteries [28].

The anti-oxidant properties of carvedilol relative to selective beta-1 blockade have been shown in different preclinical models [29-31], and contribute to its antiapoptotic effect compared to metoproplol. One mechanism here may relate to cellular calcium overload, which precedes apoptosis and is delayed by carvedilol, but not by beta-1 selective or non-selective drugs, including metoprolol and propranolol. However, similar protective effects are found when metoprolol is combined with the anti-oxidant $\mathrm{N}$-acetyl-L-cysteine [30]. In isolated rat hearts calcium overload increased mitochondrial oxygen consumption and oxygen radical production. Both were suppressed by carvedilol, but not by metoprolol [31].

In another study in myocardial tissue from patients with end-stage heart failure carvedilol resulted in better cardiac energy conservation than beta-1 selective blockade with significantly less myofibrillar tension-dependent ATP consumption with chronic treatment with carvedilol as compared to metoprolol [32]. Furthermore, whereas in heart failure post-MI beta-blockade up-regulates reduced SERCA levels and improves cardiac remodeling and impaired cardiac function, these effects are more pronounced with carvedilol than metoprolol [33].

\section{Carvedilol as preferred beta-blocker in cardiac remodeling and heart failure-clinical studies}

Do the better effects of carvedilol observed in preclinical studies translate into more pronounced anti-remodeling effects in the clinical situation when compared to beta-1 selective blockade?

In heart failure patients carvedilol exerts a more potent anti-adrenergic effect than metoprolol during stress [34, 35] (Fig. 2). This may partly explain the better anti-remodeling properties of carvedilol observed in most studies which compared it to beta-1 selective blocking agents such as metoprolol.

Sanderson et al observed a greater decrease in LV volumes with carvedilol than with metoprolol in heart failure patients [36]. Metra and coworkers reported a significantly greater increase in LV ejection fraction after 1 year treatment with carvedilol compared to metoprolol [37]. In contrast, Kukin et al found similar improvements in LV ejection fraction [38]. However, this was a smaller study over a shorter period. Finally, in a meta-analysis including all available controlled trials Packer and colleagues also found a significantly greater increase in LV ejection fraction with carvedilol than with metoprolol [39]. 
Fig. 2 Comparison of the effect of carvedilol and metoprolol on total body and cardiac norepinephrine spillover in patients with heart failure. Carvedilol reduces norepinephrine spillover but metoprolol has no effect (from ref 35 with permission)

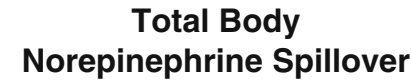

Cardiac

Norepinephrine Spillover

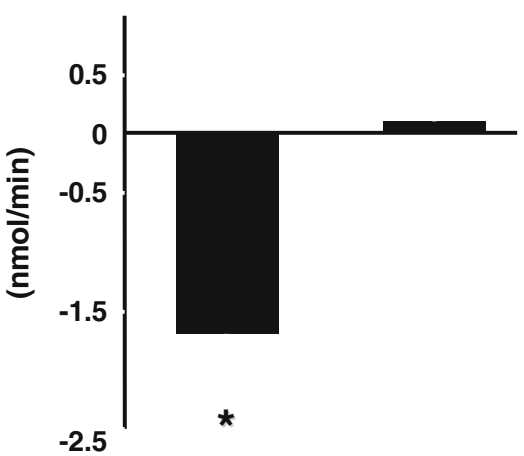

Carvedilol Metoprolol

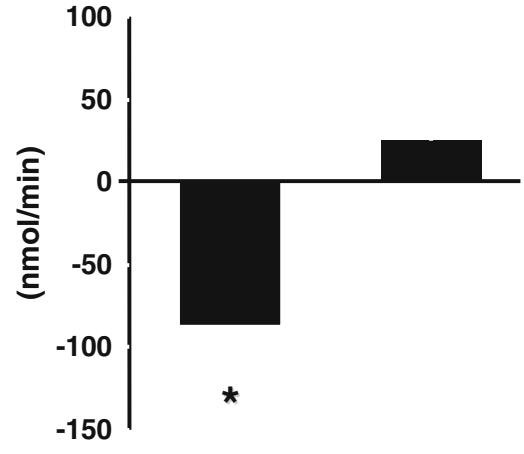

Carvedilol Metoprolol

${ }^{*} \mathbf{P}<0.05$ vs. baseline and vs. metoprolol

Taken together, the available studies did indicate a better effect of carvedilol on cardiac remodeling and cardiac function, however, none of these studies was of sufficient size or sufficient duration to allow a comparison of the long-term effects of carvedilol on morbidity and survival compared to beta-1 selective blockade.

That information was provided by the COMET study.

\section{The COMET study}

The Carvedilol or Metoprolol European Trial (COMET) evaluated the effects of carvedilol (25 mg BID) and metoprolol tartrate (50mg BID) in a randomized, doubleblind, parallel design in patients with chronic heart failure, NYHA class II-IV and LV ejection fraction $<35 \%$, on standard heart failure therapy including diuretics and ACE inhibitors [40]. To be included, patients had to be hospitalized for cardiovascular reasons in the year preceding randomization. The study was event-driven, lasting until 1,020 deaths had occurred. 3,029 patients were included, 1,511 receiving carvedilol and 1,518 metoprolol. The study duration was long, 58 months, reflecting the beneficial effects of beta-blocking agents in general in heart failure. Nevertheless, there was a clear benefit of carvedilol compared to metoprolol with a $17 \%$ greater reduction in allcause death compared to the effect of metoprolol, $p=0.0017$ (Fig. 3). It reduced annual mortality from $10.0 \%$ to $8.3 \%$ and prolonged median survival by 1.4 years compared to metoprolol. Cardiovascular deaths were reduced by $20 \%$ $(p=0.0004)$, sudden death by $19 \%(p=0.022)$, heart failure death by $13 \%(p=0.07)$ and stroke death by $66 \%(p=$ 0.0006 ), all relative to the effects of metoprolol. The greater reduction in mortality with carvedilol compared to metoprolol persisted in all pre-specified subgroups, including, age, sex, LV ejection fraction, ischemic vs non-ischemic etiology, heart rate and blood pressure at baseline. In contrast, the co-primary endpoint all-cause death and allcause hospitalisation did not differ between carvedilol and metoprolol groups, but all pre-defined secondary endpoints showed a significantly better effect of carvedilol.

After 4 months of treatment, there was a minimal, but significant difference in the effect on heart rate, 1.6 beat/ minute less in the carvedilol group. A subsequent analysis of heart rate and blood pressure at 4 months treatment did however show a consistent treatment benefit of carvedilol, irrespective of whether heart rates were below or above median heart rate and above or below median systolic blood pressure [41]. Similarly, in view of the suggestion that by using metoprolol tartrate rather than succinate, the

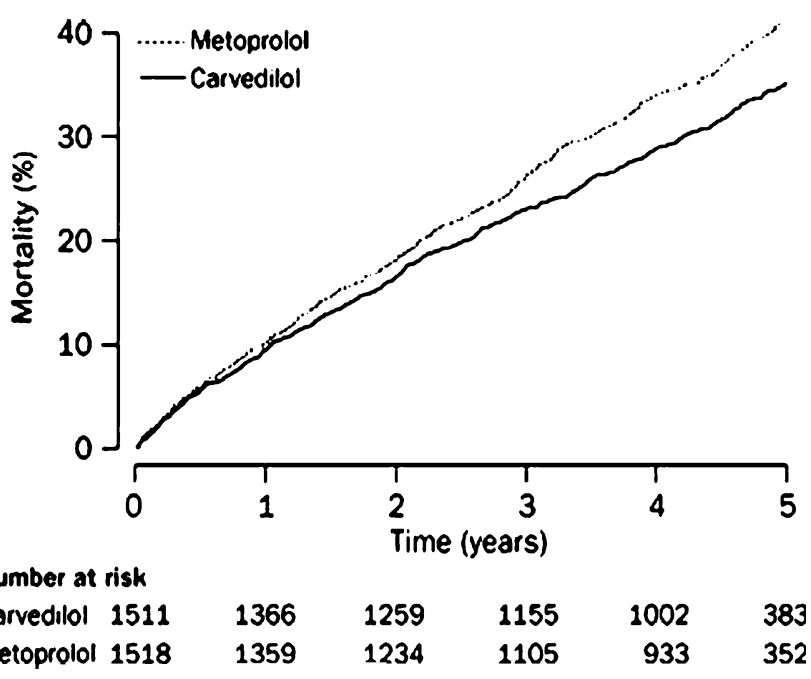

Fig. 3 Total mortality in COMET. Carvedilol significantly improves survival by $17 \%$ relative to the effect of metoprolol (from ref 40 with permission) 
dose of metoprolol could have been an issue, patients on and below target dose at 4 months were compared showing again a consistent better effect of carvedilol compared to metoprolol, irrespective of dose. The latter observation accords to previous comparative studies between metoprolol tartrate (in a similar dose as used in COMET) and metoprolol succinate, which showed a comparable effect on heart rate [42]. Moreover, the MERIT-HF trial in patients on a high dose and low dose of metoprolol succinate had the same outcome (although patients were not randomised into those groups)[43]. After myocardial infarction there was no difference in 5 year outcome between metoprolol tartrate $(41 \%$ on $100 \mathrm{mg}$ once daily, $46 \%$ on $200 \mathrm{mg}$ ) and succinate $(49 \%$ on $100 \mathrm{mg}, 28 \%$ on $200 \mathrm{mg}$ ) [44].

Thus, the results of COMET provide a clear distinction between the better clinical effects of carvedilol as compared to beta-1 selective blockers, i.c. metoprolol, in patients with chronic heart failure, and cannot be explained by differences in heart rate, blood pressure or dose.

\section{What more can we learn from COMET?}

\section{Vasculo-protective properties of carvedilol}

In COMET, several vascular endpoints were studied, including cardiovascular death, stroke, stroke death, MI and unstable angina.

MIs were reported in 69 carvedilol and 94 metoprolol patients (HR 0.71, CI 0.52=0.97, $p=0.03$ ) (Fig. 4) [45]. Cardiovascular death or non-fatal MI combined were reduced by $19 \%$ in carvedilol, compared to metoprolol $(p=0.0009)$. Unstable angina was reported as an adverse event by $29 \%$ less carvedilol patients (0.049). Also, strokes were $21 \%$ less in the carvedilol group, and a stroke or MI combined in $25 \%$ less carvedilol patients $(=0.015)$. Fatal MI or fatal stroke were reported in 34 carvedilol and in 72 metoprolol patients (HR 0.46, CI 0.31-0.69, $p=0.0002$ ). Also, death following a non-fatal MI or stroke occurred significantly less in the carvedilol group $(37 \%, p=0.0086)$. Of importance, these effects were generally observed early in the study. It can be concluded that carvedilol improves vascular outcomes more than metoprolol and that these results suggest a ubiquitous protective effect of carvedilol against major vascular events.

This effect is probably dependent on different mechanisms.

The vascular endothelium contains both beta- 1 and -2 as well as alpha-1 receptors. Blockade of all 3 adrenergic receptors by carvedilol provides for better endotheliumdependent vasodilatation than more selective beta-blockers [46]. Carvedilol, but not metoprolol results in vasodilatation and better improves endothelial function [47]. Antioxidative properties of carvedilol may contribute to improving free-radical induced endothelial dysfunction,
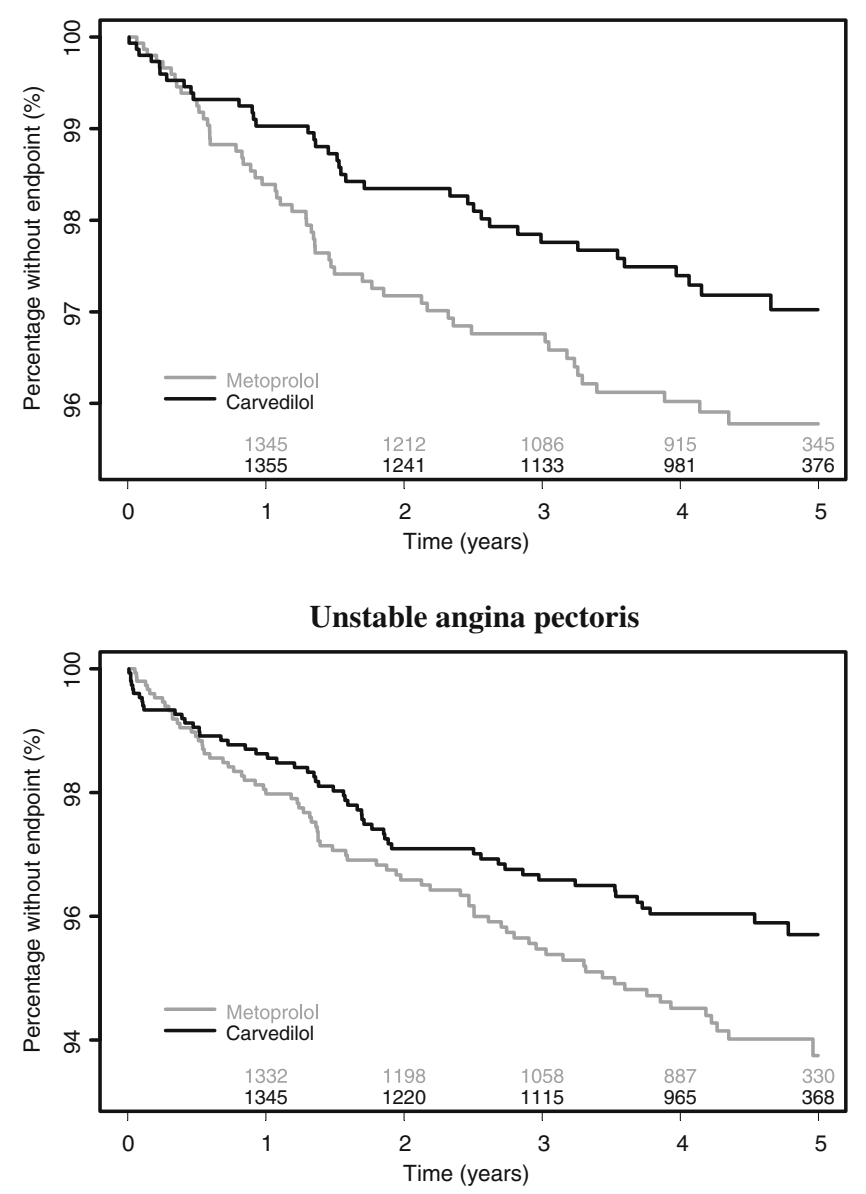

Fig. 4 Vasculoprotective effects of carvedilol in COMET. Carvedilol significantly reduces acute myocardial infarction and unstable angina by $29 \%$ compared to metoprolol

whereas anti-apoptotic effects could contribute to a reduction of myocardial injury and infarct size following ischemia-reperfusion [48-55]. These properties may also affect atherosclerosis formation [56].

Finally, carvedilol, suppresses norepinephrine release from the ischemic heart, which is likely to contribute to better anti-ischemic effects, vaso-dynamics and, possibly vasculo-protection by the drug [57].

\section{Carvedilol and diabetes mellitus}

In COMET, diabetic events occurred in $22 \%$ less carvedilol patients than in metoprolol patients $(p=0.039)$ [58]. New onset diabetes was diagnosed in $10.4 \%$ versus $12.6 \%$ cases in the carvedilol and metoprolol treatment groups, respectively (HR 0.78, $p=0.048$ ). Patients with diabetes at baseline had an increased mortality, compared to nondiabetics $(45.3 \%$ versus, $33.9 \%)$. Both diabetics and nondiabetics at baseline had a similar reduction in mortality with carvedilol compared to metoprolol $(15 \%$ and $18 \%$, respectively). Thus, whereas there was a high prevalence and incidence of diabetes in COMET patients, new onset 
diabetes was more likely to occur during treatment with metoprolol than during treatment with carvedilol.

In the Gemini study, in patients with diabetes and hypertension, in patients not taking insulin sensitizers, metoprolol tartrate significantly worsened insulin resistance, an effect not seen with carvedilol [59]. Similarly, in patients after an MI, carvedilol improved insulin resistance [60]. In the latter study, carvedilol also reduced total cholesterol and LDL levels to a greater extent than metoprolol. Reductions in triglycerides, total cholesterol and non-HDL levels were also reported to be significantly greater with carvedilol than with metoprolol in the GEMINI study [61], whereas a different report of the latter study indicated more weight gain with metoprolol than carvedilol [62].

Taken together, carvedilol may also be a better choice than metoprolol in heart failure in combination with determinants of the metabolic syndrome.

\section{Which is the preferred beta-blocker in patients with chronic obstructive lung disease?}

The respiratory system is characterized by a significant predominance of beta-2 receptors, particularly present in the alveoli, where they regulate gas exchange efficacy. Obviously, beta-1 selective blockers may have a different effect than carvedilol. Recent studies indicate that a beta-1 selective agent will result in a slightly higher peak VO2 than carvedilol, although probably not clinically relevant, except in patients with a low lung diffusion (DLco). Carvedilol reduces DLco to some extent, whereas bisoprolol does not, in heart failure patients [63].

Previous studies by the same group indicate that carvedilol has no effect on lung volumes and DLco in patients with heart failure without obstructive airway disease [64]. Another study suggests that a sizeable number of HF patients with COPD or asthma tolerate carvedilol for a relatively long period [65]. Part of this may be due to the concomitant alpha-adrenergic blocking properties and resulting bronchodilatation of carvedilol, although this is as yet speculative. Beta-blockers are not contra-indicated in the presence of lung function impairment, but their use should be guided by lung function tests and knowledge of the pharmacological properties of the specific beta-blocker [66]. In any case, it would appear wise to choose a beta1selective blocker in patients with reversible obstructive airway disease or during exacerbation of COPD.

\section{Conclusion}

There is ample evidence from pre-clinical and clinical studies that carvedilol has more pronounced anti-remodeling effects in heart failure than selective beta-blocking agents, more specifically the beta-1 selective blocking drugs metoprolol and bisoprolol.

The significant difference in efficacy is due to a combination of factors, including the comprehensive blockade of all 3 adrenergic receptors by carvedilol, and its anti-oxidative, antiapoptotic and anti-endothelin effects.

As a result, in the COMET study carvedilol led to a significant and clinically relevant improvement of survival, better well-being, less new onset diabetes and significantly less vascular events including MI and stroke.

Taken together, these results clearly indicate that carvedilol is the preferred beta-blocker in the treatment of chronic heart failure.

\section{References}

1. Packer M, Bristow MR, Cohn JN, et al. The effect of carvedilol on morbidity and mortality in patients with chronic heart failure. U.S. Carvedilol Heart Failure Study Group. N Engl J Med. 1996;334:1349-55.

2. CIBIS II Investigators and Committees. The Cardiac Insufficiency Bisoprolol Study II (CIBIS II): a randomised trial. Lancet. 1999;353:9-13.

3. Hjalmarson A, Goldstein S, Fagerberg B, et al. Effect of metoprolol $\mathrm{CR} / \mathrm{XL}$ in chronic heart failure: metoprolol $\mathrm{CR} / \mathrm{XL}$ randomized intervention trial in congestive heart failure (MERITHF). Lancet. 1999;353:2001-7.

4. Packer M, Coats AJS, Fowler MB, et al. For the Carvedilol Prospective Randomized Cumulative Survival Study Group (COPERNICUS). Effect of carvedilol on survival in severe chronic heart failure. New Engl J Med. 2001;344:1651-8.

5. The CAPRICORN Investigators. Effect of carvedilol on outcome after myocardial infarction in patients with left ventricular dysfunction: the CAPRICORN randomised trial. Lancet. 2001;357:1385-90.

6. Flather MD, Shibata MC, Coats AJ, et al. Randomized trial to determine the effect of nebivolol on mortality and cardiovascular hospital admission in elderly patients with heart failure (SENIORS). Eur Heart J. 2005;26:215-25.

7. Hunt SA, Abraham WT, Chin MH, et al. ACC/AHA 2005 guideline update for the diagnosis and management of chronic heart failure in the adult. Circulation. 2005;112:e154-235.

8. Swedberg K, Cleland J, Dargie H, et al. Guidelines for the diagnosis and treatment of chronic heart failure: executive summary (update 2005). Eur Heart J. 2005;26:1115-40.

9. Cohn JN. Remodeling as an end-point in heart failure therapy. Cardiovasc Drugs Ther. 2004;18:7-8.

10. Remme WJ. Pharmacological modulation of cardiovascular remodelling: a guide to heart failure therapy. Cardiovasc Drugs Ther. 2003;17:349-60.

11. Yamazaki T, Komuro I, Kudoh S, et al. Role of ion channels and exchangers in mechanical stretch-induced cardiomyocyte hypertrophy. Circ Res. 1998;82:430-7.

12. Ungureanu-Longrois D, Balligand JL, Simmons SW, et al. Induction of nitric oxide synthase activity by cytokines in ventricular myocytes is necessary but not sufficient to decrease contractile responsiveness to $\beta$-adrenergic agonists. Circ Res. 1995;77:494-502.

13. Li X, Moody MR, Engl D, et al. Cardiac-specific overexpression of tumor necrosis factor- $\alpha$ causes oxidative stress 
and contractile dysfunction in mouse diaphragm. Circulation. 2000;102:1690-6.

14. Francis GS, Benedict C, Johnston DE, et al. Comparison of neuroendocrine activation in patients with left ventricular dysfunction with and without congestive heart failure: a substudy of the Studies Of Left Ventricular Dysfunction (SOLVD). Circulation. 1990;82:1724-9.

15. Mann DL, Kent RL, Parsons B, Cooper G. Adrenergic effects on the biology of the adult mammalian cardiocyte. Circulation. 1992;24:549-64.

16. Communal C, Singh K, Pimentel DR, Colucci WS. Norepinephrine stimulates apoptosis in adult rat ventricular myocytes by activation of beta-adrenergic pathway. Circulation. 1998;98:1329-34.

17. Tan LB, Benjamin IJ, Clark WA. Beta-adrenergic receptor desensitization may serve a cardioprotective role. Cardiovasc Res. 1992;26:608-14.

18. Prabhu SD, Wang G, Luo J, et al. Beta-adrenergic receptor blockade modulates $\mathrm{Bcl}-\mathrm{X}(\mathrm{S})$ expression and reduces apoptosis in failing myocardium. J Mol Cell Cardiol. 2003;35:483-93.

19. Du XJ, Autelitano D, Dilley RJ, Wang B, Dart AM, Woodcock EA. $\beta_{2}$-adrenergic overexpression exacerbates development of heart failure after aortic stenosis. Circulation. 2000;101:71-7.

20. Billman GE, Castillo LC, Hensley J, Hohl CM, Altschuld RA. $\beta_{2}$-adrenergic receptor antagonists protect against ventricular fibrillation. In vivo and in vitro evidence for enhanced sensitivity to $\beta_{2}$-adrenergic stimulation in animals susceptible to sudden death. Circulation. 1997;96:1914-22.

21. Molenaar P, Christ T, Ravens U, Kaumann A. Carvedilol blocks beta 2 - more than beta-1 adrenoceptors in the heart. Cardiovasc Res. 2006;69:128-39.

22. Kindermann M, Maack C, Schaller S, et al. Carvedilol but not metoprolol reduces $\beta$-adrenergic responsiveness after complete elimination from plasma in vivo. Circulation. 2004;109:3182-9.

23. Zhao Q, Wu T-G, Jiang Z-F, Chen G-W, Lin Y, Wang L-X. Effect of $\beta$-blockers on $\beta_{3}$-adrenoceptor expression in chronic heart failure. Cardiovasc Drugs Ther. 2007;21:85-90.

24. Wisler JW, DeWire SM, Whalen EJ, et al. A unique mechanism of $\beta$-blocker action: carvedilol stimulates $\beta$-arrestin signaling. Proc Natl Acad Sci. 2007;104:16657-62.

25. Nicolaides LA, Poornima I, Parikh P, Magovern M, Shen YT, Shannon RP. The effects of combined versus selective adrenergic blockade on left ventricular and systemic hemodynamics, myocardial substrate preference, and regional perfusion in conscious dogs with dilated cardiomyopathy. J Am Coll Cardiol. 2006;47:1871-81.

26. Hanada K, Asari K, Saito M, Kawana J, Mita M, Ogata H. Comparison of pharmacodynamics between carvedilol and metoprolol in rats with isoproterenol-induced cardiac hypertrophy: effects of enantiomeres. Eur J Pharmacol. 2008;589:194-200.

27. Lai K-B, Sanderson JE, Yu C-M. Suppression of collagen production in norepinephrine stimulated cardiac fibroblasts culture: differential effect of $\alpha$ and $\beta$-adrenoceptor antagonism. Cardiovasc Drugs Ther. 2009;23:271-80.

28. Batenburg WW, van Esch JH, Garrelds IM, et al. Carvedilolinduced antagonism of angiotensin II: a matter of alpha-1 adrenoceptor blockade. J Hypertens. 2006;24:1355-63.

29. Bartholomeu JB, Vanzelli AS, Rolim NP, et al. Intracellular mechanisms of specific beta-adrenoceptor antagonists involved in improved cardiac function and survival in a genetic model of heart failure. J Mol Cell Cardiol. 2008;45:240-9.

30. Wang R, Miura T, Hanada N, et al. Pleiotropic effects of the betaadrenoceptor blocker carvedilol on calcium regulation during oxidative stress-induced apoptosis in cardiomyocytes. J Pharmacol Exp Ther. 2006;318:45-52.

31. Kametani R, Miura T, Harada N, et al. Carvedilol inhibits mitochondrial oxygen consumption and superoxide production during calcium overload in isolated heart mitochondria. Circ J. 2006;70:321-6.

32. Brixius $\mathrm{K}, \mathrm{Lu} \mathrm{R}$, Boelck $\mathrm{B}$, et al. Chronic treatment with carvedilol improves $\mathrm{Ca}(2+)$-dependent ATP consumption in triton $\mathrm{X}$-skinned fiber preparations of human myocardium. J Pharmacol Exp Ther. 2007;322:222-7.

33. Sun YL, Hu SJ, Wang LH, et al. Effect of beta-blockers on cardiac function and calcium handling protein in postinfarction heart failure rats. Chest. 2005;128:1812-21.

34. Kohno T, Yoshikawa T, Yoshizawa A, et al. Carvedilol exerts more potent antiadrenergics effect than metoprolol in heart failure. Cardiovasc Drugs Ther. 2005;19:347-55.

35. Azevedo ER, Kubo T, Mak S, et al. Non-selective versus selective $\beta$ adrenergic receptor blockade in congestive heart failure: differential effects on sympathetic activity. Circulation. 2001;104:2194-9.

36. Sanderson JE, Chan SKW, Yip G, et al. Beta-blockade in heart failure. A comparison of carvedilol with metoprolol. J Am Coll Cardiol. 1999;34:1522-8.

37. Metra M, Gibbini R, Nodari S, Boldi E, Modena MG, Dei Cas L. Differential effects of beta-blockers in patients with heart failure. A prospective, randomized, double-blind comparison of the long-term effects of metoprolol versus carvedilol. Circulation. 2000;102:546-51.

38. Kukin ML, Kalman J, Charney Rh, et al. Prospective, randomized comparison of effect of long-term treatment with metoprolol or carvedilol on symptoms, exercise, ejection fraction, and oxidative stress in heart failure. Circulation. 1999;99:2645-51.

39. Packer M, Antonopoulos GV, Berlin JA, Chittams J, Konstam MA, Udelson JE. Comparative effects of carvedilol and metoprolol on left ventricular ejection fraction in heart failure: results of a meta-analysis. Am Heart J. 2001;141:899-907.

40. Poole-Wilson PA, Swedberg K, Cleland JGF, et al. Comparison of carvedilol and metoprolol on clinical outcomes in patients with chronic heart failure. Results of the Carvedilol Or Metoprolol European Trial (COMET). Lancet. 2003;362:7-13.

41. Metra M, Torp-Pedersen C, Swedberg K, et al. Influence of heart rate, blood pressure, and beta-blocker dose on outcome and the differences in outcome between carvedilol and metoprolol tartrate in patients with chronic heart failure: results of the COMET trial. Eur Heart J. 2005;26:2259-68.

42. Kukin ML, Mannino MM, Freudenberger RS, Kalman J, BuchholzVarley C, Ocampo O. Hemodynamic comparison of twice daily metoprolol tartrate with once daily metoprolol succinate in congestive heart failure. J Am Coll Cardiol. 2000;35:45-50.

43. Wikstrand J, Hjalmarson A, Waagstein F, et al. MERIT-HF Study Group. Dose of metoprolol CR/XL and clinical outcomes in patients with heart failure: Analysis of the experience in metoprolol $\mathrm{CR} / \mathrm{XL}$ randomized intervention trial in chronic heart failure (MERIT-HF). J Am Coll Cardiol. 2002;40:491-8.

44. Herlitz J, Dellborg M, Karlson BW, et al. Similar risk reduction of death of extended-release metoprolol once daily and immediaterelease metoprolol twice daily during 5 years after myocardial infarction. Cardiovasc Drugs Ther. 1999;13:127-35.

45. Remme WJ, Torp-Pedersen C, Cleland JGF, et al. Carvedilol protects better against vascular events than metoprolol in heart failure. Results from COMET. J Am Coll Cardiol. 2007;49:963-71.

46. Von zur Muhlen B, Millgard J, Lind L. Divergent effects of different beta-blocking agents on endothelin-dependent vasodilatation in the human forearm. Blood Press. 2000;9:287-92.

47. Intengan HD, Schiffrin EL. Disparate effects of carvedilol versus metoprolol treatment of stroke-prone spontaneously hypertensive rats on endothelial function of resistance arteries. J Cardiovasc Pharmacol. 2000;35:763-8.

48. Matsuda Y, Akita H, Terashima M, Shiga N, Kanazawa K, Yokoyama M. Carvedilol improves endothelium-dependent dilatation in patients with coronary artery disease. Am Heart J. 2000;140:753-9. 
49. Lopez BL, Christopher TA, Yue TL, Ruffolo R, Feuerstein GZ, Ma XL. Carvedilol, a new beta-adrenoceptor blocker antihypertensive drug, protects against free-radical-induced endothelial dysfunction. Pharmacology. 1995;51:165-73.

50. Gao F, Chen J, Lopez BL, et al. Comparison of bisoprolol and carvedilol cardioprotection in a rabbit ischemia and reperfusion model. Eur J Pharmacol. 2000;406:109-16.

51. Ma XL, Yue TL, Lopez BL, et al. Carvedilol, a new betaadrenoceptor blocker and free radical scavenger attenuates myocardial ischemia reperfusion injury in hypercholesterolemic rabbits. J Pharmacol Exp Ther. 1996;277:128-36.

52. Oliveira PJ, Rolo AP, Monteiro P, Goncalves L, Palmeira CM, Moreno AJ. Impact of carvedilol on the mitochondrial damage induced by hypxanthine and xantine oxidase-what role in myocardial ischemia and reperfusion? Rev Port Cardiol. 2002;21:1447-55.

53. Asanuma H, Minamino T, Sanada S, et al. Beta-adrenoceptor blocker carvedilol provides cardioprotection via an adenosine-dependent mechanism in ischemic canine hearts. Circulation. 2004;109:2773-9.

54. Romeo F, Li D, Shi M, Mehta JL. Carvedilol prevents epinephrine-induced apoptosis in human coronary artery endothelial cells: modulation of Fas/Fas ligand and caspase-3 pathway. Cardiovasc Res. 2000;45:788-94.

55. Rossig L, Haendeler J, Mallat Z, et al. Congestive heart failure induces endothelial cell apoptosis: protective role of carvedilol. J Am Coll Cardiol. 2000;36:2081-9.

56. Chen JW, Lin FY, Chen YH, Wu T, Chen YL, Lin SJ. Carvedilol inhibits tumor necrosis factor-alpha-induced endothelial transcription factor activation, adhesion molecule expression and adhesiveness to human mononuclear cells. Arterioscler Thromb Vasc Biol. 2004;24:2075-81.
57. Kurz T, Richardt D, Gorge B, et al. Differential effects of carvedilol on norepinephrine release in normoxic and ischemic heart. J Cardiovasc Pharmacol. 2000;36:96-100.

58. Torp-Pedersen C, Metra M, Charlesworth A, et al. Effects of metoprolol and carvedilol on pre-existing and new onset diabetes in patients with chronic heart failure: data from the Carvedilol Or Metoprolol European Trial (COMET). Heart. 2007;93:968-73.

59. Fonseca V, Bakris GL, Bell DSH, et al. Differential effect of betablocker therapy on insulin resistance as a function of insulin sensitizer use: results of GEMINI. Diabet Med. 2007;24:759-63.

60. Basat O, Ucak S, Seber S, Oztekin E, Altuntas Y. After myocardial infarction carvedilol improves insulin resistance compared to metoprolol. Clin Res Cardiol. 2006;95:99-104.

61. Bell DSH, Bakris GL, McGill JB. Comparison of carvedilol and metoprolol on serum lipid concentration in diabetic hypertensive patients. Diab Obes Metab. 2009;11:234-8.

62. Messerli FH, Bell DSH, Fonseca V, et al. Body weight changes with beta-blocker use: results of GEMINI. Am J Med. 2007;120:10-5.

63. Agostoni P, Contini M, Cattadori G, et al. Lung function with carvedilol and bisoprolol in chronic heart failure: Is $\beta$ selectivity relevant? Eur J Heart Fail. 2007;9:827-33.

64. Guazzi M, Agostoni P, Maturri M, Pontone G, Guazzi MD. Pulmonary function, cardiac function and exercise capacity in a follow-up of patients with congestive heart failure treated with carvedilol. Am Heart J. 1999;138:460-7.

65. Kotlyar E, Keogh AM, Macdonald PS, Arnold RH, McCaffrey DJ, Glanville AR. Tolerability of carvedilol in patients with heart failure and concomitant chronic obstructive pulmonary disease or asthma. J Heart Lung Transplant. 2002;21:1290-5.

66. Agostoni P, Palermo P, Contini M. Respiratory effects of $\beta$-blocker therapy in heart failure. Cardiovasc Drugs Ther. 2009;23:377-84. 